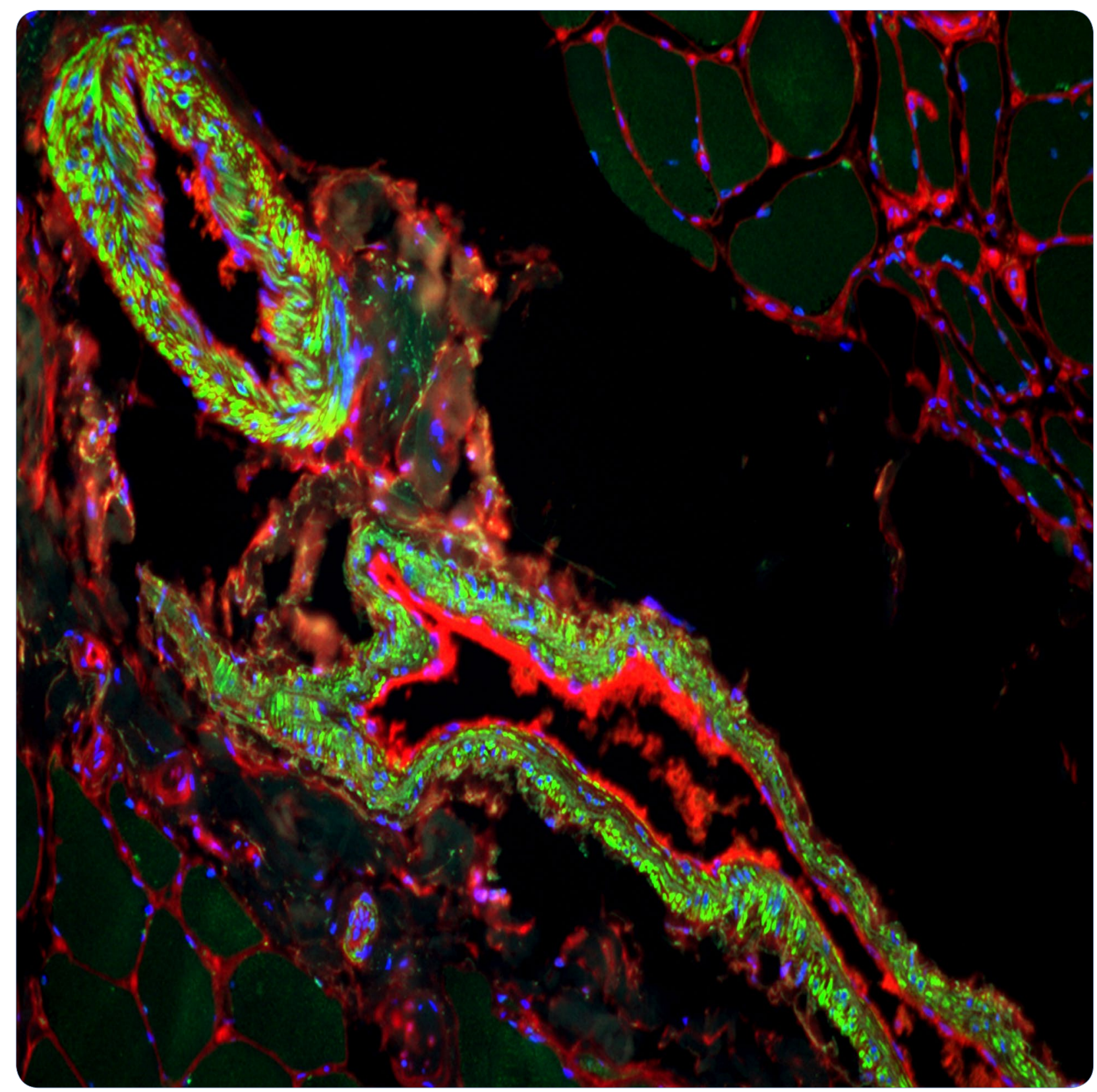

Transforming growth factor-beta 1 produced by vascular smooth muscle cells predicts fibrosis in the gastrocnemius of patients with peripheral artery disease

Ha et al. 


\title{
Transforming growth factor-beta 1 produced by vascular smooth muscle cells predicts fibrosis in the gastrocnemius of patients with peripheral artery disease
}

Duy M. Ha ${ }^{1,2}$, Lauren C. Carpenter ${ }^{1}$, Panagiotis Koutakis' ${ }^{1}$, Stanley A. Swanson ${ }^{1}$, Zhen Zhu' ${ }^{1}$, Mina Hanna ${ }^{2}$, Holly K. DeSpiegelaere ${ }^{3}$, Iraklis I. Pipinos ${ }^{1,2,3,5^{*}+}$ and George P. Casale ${ }^{1,4^{*}+}$

\begin{abstract}
Background: Lower leg ischemia, myopathy, and limb dysfunction are distinguishing features of peripheral artery disease (PAD). The myopathy of PAD is characterized by myofiber degeneration in association with extracellular matrix expansion, and increased expression of transforming growth factor-beta 1 (TGF- $\beta 1$; a pro-fibrotic cytokine). In this study, we evaluated cellular expression of TGF- $\beta 1$ in gastrocnemius of control (CTRL) and PAD patients and its relationship to deposited collagen, fibroblast accumulation and limb hemodynamics.

Methods: Gastrocnemius biopsies were collected from PAD patients with claudication (PAD-II; N = 25) and tissue loss (PAD-IV; $N=20$ ) and from CTRL patients $(N=20)$. TGF- $\beta 1$ in slide-mounted specimens was labeled with fluorescent antibodies and analyzed by quantitative wide-field, fluorescence microscopy. We evaluated co-localization of TGF- $\beta 1$ with vascular smooth muscle cells (SMC) (high molecular weight caldesmon), fibroblasts (TE-7 antigen), macrophages (CD163), T cells (CD3) and endothelial cells (CD31). Collagen was stained with Masson Trichrome and collagen density was determined by quantitative bright-field microscopy with multi-spectral imaging.

Results: Collagen density increased from CTRL to PAD-II to PAD-IV specimens (all differences $p<0.05$ ) and was prominent around microvessels. TGF- $\beta 1$ expression increased with advancing disease (all differences $p<0.05$ ), correlated with collagen density across all specimens $(r=0.864 ; p<0.001)$, associated with fibroblast accumulation, and was observed exclusively in SMC. TGF- $\beta 1$ expression inversely correlated with ankle-brachial index across PAD patients $(r=-0.698 ; p<0.001)$.
\end{abstract}

Conclusions: Our findings support a progressive fibrosis in the gastrocnemius of PAD patients that is caused by elevated TGF- $\beta 1$ production in the SMC of microvessels in response to tissue hypoxia.

Keywords: Peripheral artery disease, Skeletal muscle, Fibrosis, Transforming growth factor-beta 1, Vascular smooth muscle cells, Microvasculature

\section{Background}

Lower leg ischemia, myopathy and limb dysfunction are distinguishing features of peripheral artery disease

\footnotetext{
*Correspondence: ipipinos@unmc.edu; gpcasale@unmc.edu ${ }^{\dagger}$ Iraklis I. Pipinos and George P. Casale are co-senior authors

${ }^{1}$ Department of Surgery, University of Nebraska Medical Center, Omaha, NE, USA

Full list of author information is available at the end of the article
}

(PAD) caused by atherosclerotic blockages of the arteries supplying the legs. This disease affects over 27 million people in North America and Europe, where the prevalence is estimated to be $16 \%$ of individuals 55 years and older, and is associated with a 5 -year mortality rate of as high as $30 \%$ [1-3]. Overall, about 11 million people with PAD are symptomatic $[1,4]$ and the majority experience claudication, i.e., walking-induced leg muscle pain that is relieved by rest (Fontaine Stage II). At later stages 
of the disease, PAD patients experience foot pain at rest (Fontaine Stage III) and non-healing ulcers, necrosis, and gangrene in the affected limb (Fontaine Stage IV). Hemodynamic changes were thought to be the sole cause of these symptoms [5-7]. However, we and others have demonstrated that a myopathy exists in the lower legs of PAD patients and contributes to limb dysfunction [8-13].

Myopathy in the affected legs of PAD patients is characterized by myofiber degeneration, elevated pro-fibrotic cytokines, and increased fibrosis [11, 14-18]. In a recent study, we found an average three-fold increase of transforming growth factor-beta 1 (TGF- $\beta 1$; a potent inducer of fibrosis) in homogenates of gastrocnemius biopsies from PAD patients compared to controls. TGF- $\beta 1$ was increased in the biopsies of all PAD patients, suggesting a chronic pro-fibrotic response [18]. Chronically elevated TGF- $\beta 1$ is known to activate myofibroblasts to deposit extracellular matrix around the myofibers and associated microvessels which interferes with oxygen and nutrient delivery, producing myofiber degeneration [19-22]. Additionally, TGF- $\beta 1$ can promote PAD myopathy by inducing myoblasts to differentiate into myofibroblasts rather than new myofibers [19-22].

TGF- $\beta 1$ may be produced by immune cells and is known to induce pathological fibrosis [19, 20, 23], but other cells have also been reported to upregulate TGF$\beta 1$ expression and contribute to fibrosis [24-28]. During wound healing, TGF- $\beta 1$ is released by immune cells to initiate the resolution phase of inflammation and to activate myofibroblasts to deposit extracellular matrix, of which a major component is collagen [29, 30]. It has been reported for various vascular diseases, including pulmonary arterial hypertension $(\mathrm{PAH})$, that vascular smooth muscle cells (SMC) transition from a contractile to a synthetic phenotype that produces TGF- $\beta 1$ [31-33]. In $\mathrm{PAH}$, chronic hypoxia induces substantial thickening of vascular intima, media and adventitia caused by extensive proliferation and hypertrophy of vascular SMC that exhibit increased expression of TGF- $\beta 1$, and deposition of extracellular matrix; features that extend to normally non-muscularized microvessels [34, 35]. In PAD, a similar transitioning of vascular SMC may occur in response to exercise-induced ischemia and the chronic intermittent hypoxia that are caused by atherosclerosis of the large vessels supplying the legs. If that is true, we expect this transitioning to also be related to the degree of hemodynamic compromise of the affected extremity and the clinical stage of PAD. In this study, we evaluated the cellular expression of TGF- $\beta 1$ in the gastrocnemius of control and PAD patients with advancing Fontaine Stage, and its relationship to collagen deposition, fibroblast accumulation, and Ankle-Brachial Index (ABI).

\section{Methods}

\section{Human subjects}

The experimental protocol was approved by the Institutional Review Boards of the Veterans Affairs NebraskaWestern Iowa and University of Nebraska Medical Centers. All subjects gave informed consent.

\section{PAD groups}

We recruited 25 patients with claudication (Fontaine Stage II, PAD-II) and 20 patients presenting with tissue loss (Fontaine Stage IV, PAD-IV) who were undergoing lower extremity operations for symptomatic PAD. The diagnosis for each PAD patient was established on the basis of medical history, physical examination, decreased ankle brachial index (ABI < 0.9), and computerized or standard arteriography that revealed stenotic and/or occluded arteries supplying the lower extremities. PAD-II patients presented with intermittent claudication, but no rest pain or tissue loss. PADIV patients presented with non-healing ulcers and/or gangrene.

\section{Control group}

We recruited 20 control patients (CTRL) who were undergoing lower extremity operations for indications other than PAD. Control patients led sedentary lifestyles and had no history of PAD symptoms. CTRL patients had normal blood flow to their lower limbs as indicated by normal lower extremity pulses at examination and normal ABI at rest and after stress.

\section{Biopsy}

Gastrocnemius samples weighing approximately $250 \mathrm{mg}$ were obtained from the anteromedial aspect of the muscle belly, $10 \mathrm{~cm}$ distal to the tibial tuberosity. All biopsies were obtained with a $6 \mathrm{~mm}$ Bergstrom needle. Some samples were frozen in liquid nitrogen for biochemical analysis while others were placed immediately into cold methacarn. After $48 \mathrm{~h}$ in methacarn, the specimens were transferred to cold $50 \%$ ethanol, and subsequently embedded in paraffin.

\section{Quantitative assessment of collagen \\ Masson Trichrome stain}

Paraffin-embedded biopsies sectioned at 4 microns were stained with a Masson Trichrome Kit according to manufacturer's protocol (Fisher Scientific Richard Allan Scientific \#22-110-648, Pittsburg, PA, USA). Briefly, slide specimens were deparaffinized in xylene, hydrated and then incubated at room temperature in Bouin's fixative for $16 \mathrm{~h}$. Nuclei were stained with Weigert's Hematoxylin, myofiber cytoplasm with Scarlet Red, and collagen with 
Aniline Blue dye, with washes in-between steps. Stained slide specimens were dehydrated and mounted with Permount (Fisher Scientific \#SP15-100, Pittsburg, PA, USA).

\section{Image acquisition and spectral analysis}

Microscopic fields of the five most fibrotic regions were collected from each Masson Trichrome stained slide ( $20 \times$ objective). Muscle tissue completely filled each chosen microscopic field. Imaging was implemented by multispectral wide-field microscopy, using a Leica microscope (North Central Instruments DMRXA2 Model, Plymouth, MN, USA) coupled with the Nuance EX Multispectral Imaging System (PerkinElmer N-MSI-EX Model, Waltham, MA, USA) that incorporates a CCD camera and liquid crystal tunable filter. This system generates an absorbance spectrum at each pixel of a twodimensional spatial image of the specimen. The Nuance software quantitatively extracts the grey scale image of deposited collagen, which is then transferred to the Image-Pro ${ }^{\circledR}$ Plus image analysis software (Media Cybernetics, Warrendale, PA, USA) for quantification of collagen area and density. Collagen density was determined as area-weighted mean pixel intensity (12-bit grey scale). Quantitative analysis was performed blinded from the group status.

\section{Validation of spectral analysis}

Spectral analysis was validated by the hydroxyproline assay for collagen content in whole muscle. Total collagen content of human gastrocnemius specimens was measured by a hydroxyproline assay based on a previously established protocol for skeletal muscle [36]. To determine intersession reliability, slide specimens of biopsy samples from ten patients were stained by Masson's Trichrome in two separate analytical sessions and assessed by spectral analysis. Detailed methodologies and validation results can be found in "Additional file 1".

\section{Quantitative fluorescence microscopy}

We describe a novel application of quantitative fluorescence microscopy (QFM), a technique developed by our laboratory that has previously been validated using multiple biomarkers of PAD and other diseases, such as prostate cancer [17, 37-41].

\section{Quantitative measurement of TGF- $\beta 1$ expression}

Duplicate slide specimens of gastrocnemius biopsies were exposed to a rabbit anti-TGF- $\beta 1$ antibody (Abcam Ab53169, Cambridge, MA, USA) and a mouse antiCD31 antibody (Abcam Ab9498, Cambridge, MA, USA; to validate identification of microvessels). Slides were then treated with both goat anti-rabbit IgG secondary antibody coupled with Alexa Fluor ${ }^{\circledR} 555$ and goat antimouse IgG secondary antibody conjugated with Alexa Fluor $^{\circledR} 488$ (Life Technologies \#A21429 and \#A11029, Carlsbad, CA, USA). Isotype control slides were treated with rabbit IgG (Vector Laboratories \#I-1000, Burlingame, CA, USA) and mouse IgG1 (eBioscience \#14-4714-85, San Diego, CA, USA) at the same concentration as the anti-TGF- $\beta 1$ and anti-CD31 antibodies, respectively. For epitope recovery, Tris buffer $(\mathrm{pH}$ 9.0) was used. All labeling procedures were performed with a fully programmable, robotic autostainer (BioGenex i6000 Model, Fremont, CA, USA). The labeled specimens were mounted in ProLong Gold ${ }^{\circledR}$ anti-fade medium containing 4,6-Diamidino-2-phenylindole (DAPI; a nuclear stain) (Life Technologies \#P36931, Carlsbad, CA, USA). Fluorescence images were captured with a Leica epifluorescence wide-field microscope $(10 \times$ objective) (North Central Instruments, DMRXA2 Model, Plymouth, MN, USA) and CCD camera (Hamamatsu Photonics, Orca C4742 Model, Bridgewater, NJ, USA), with Hamamatsu software (HCImage 4.0). All fields of each specimen are captured and a montage of the gray scale images was generated for analysis with Image-Pro ${ }^{\circledR}$ Plus (Media Cybernetics, Bethesda, MD, USA). TGF- $\beta 1$ positive events in the microvasculature are partitioned and both event area and mean pixel intensity are determined for each (12-bit grey scale). The sum of the products of area and mean pixel intensity of all positive events per microscopic field was computed and normalized to the total area of muscle tissue specimen analyzed. Quantitative analysis was performed blinded from the group status.

\section{Validation of quantitative fluorescence microscopy}

QFM measurements of TGF- $\beta 1$ were validated by comparing results with ELISA and qPCR measurements of muscle homogenates from PAD-II and CTRL patients $(\mathrm{N}=13$ in each group). TGF- $\beta 1$ protein expression was measured as part of a customized Human Inflammatory Cytokines Multi-Analyte ELISArray Kit (Qiagen, Valencia, CA, USA).

To measure TGF- $\beta 1$ gene transcripts in skeletal muscle biopsies, RNA extraction, reverse transcription reactions, and qPCR were performed as previously described $[40,42]$ and levels were normalized to myosin gene transcripts. Intrasession reliability was determined by comparing the TGF- $\beta 1$ measurement of each slide from the mean of its duplicate pair. Intersession reliability was determined from the averages of patient biopsy specimens, analyzed a second time in the next analytical session. Detailed methodologies and validation results can be found in "Additional file 1". 


\section{Co-localization of TGF- $\beta 1$ and Ki-67 to candidate cells by immunofluorescence}

Individual slides were treated with primary anti-TGF- $\beta 1$ antibody (Abcam Ab53169, Cambridge, MA, USA) and primary antibody specific for each of the following cell types. For endothelia, we used an antibody against CD31 (1:50 dilution) (Abcam Ab9498, Cambridge, MA, USA); for vascular smooth muscle cells, an antibody against high molecular weight caldesmon (1 ug/mL) (Abcam Ab1826, Cambridge, MA, USA); for fibroblasts, an antibody for TE-7 (1:10 dilution) (Millipore CBL271, Billerica, MA, USA); for macrophages, antibodies for CD163 (2.5 ug/mL) (Abcam Ab156769, Cambridge MA, USA) and CD68 (1 ug/mL) (Fisher Scientific Thermo MS-397, Pittsburg, PA, USA); for T cells, an antibody for CD3 (1ug/mL) (Abcam Ab699, Cambridge, MA, USA). To detect proliferative cells, we labeled with an antibody against Ki-67 (5 ug/mL) (Abcam Ab15580, Cambridge, MA, USA) and ProLong Gold ${ }^{\circledR}$ anti-fade medium with DAPI nuclear stain (Life Technologies \#P36931, Carlsbad, CA, USA). For all labels, we used Tris buffer $(\mathrm{pH}$ 9.0) for epitope recovery, except for Ki-67 where we used citrate buffer ( $\mathrm{pH}$ 6.0). Isotype control slides were stained with rabbit IgG (Vector Laboratories \#I-1000, Burlingame, CA, USA) or mouse IgG1 (eBioscience \#144714-85, San Diego, CA, USA), IgG2a (eBioscience \#144724-85, San Diego, CA, USA), or IgG2b (eBioscience \#14-4732-85, San Diego, CA, USA) at the same concentration as their respective primary antibodies. Primary antibodies to TGF- $\beta 1$ and Ki-67 were labeled with a goat anti-rabbit IgG secondary antibody coupled with Alexa Fluor $^{\circledR} 555$ (Life Technologies \#A21429, Carlsbad, CA, USA) and all other primary antibodies were labeled with a goat anti-mouse IgG secondary antibody coupled with Alexa Fluor ${ }^{\circledR} 647$ (Life Technologies \#A21236, Carlsbad, CA, USA). All labeling procedures were performed with a fully programmable, robotic autostainer (BioGenex i6000 Model, Fremont, CA, USA).

\section{Detection of fibroblast accumulation by immunohistochemistry}

Duplicate slide specimens were deparaffinized and heated in Tris buffer ( $\mathrm{pH}$ 9.0) for epitope recovery. Specimens were blocked with $10 \%$ goat serum for $5 \mathrm{~min}$, treated with primary antibody against TE-7 (1:10 dilution) (Millipore CBL271, Billerica, MA, USA), a highly specific marker of fibroblasts [43-45], and then incubated overnight for $14 \mathrm{~h}$. An isotype control treated with the same concentration of mouse IgG1 (EBioscience \#144714-85, San Diego, CA, USA) was included with each duplicate antibody-treated slide. Specimens were treated with peroxidase-conjugated secondary antibody according to instructions from the DAB Polink 2 Kit (GBI Labs
\#D22-60D, Bothell, WA, USA). Hematoxylin (Fisher Scientific Richard Allan Scientific \#72511, Pittsburg, PA, USA) was used as the counterstain for $4 \mathrm{~min}$. Slides were dehydrated and mounted in Permount (Fisher Scientific \#SP15-100, Pittsburg, PA, USA). Staining was implemented with a fully programmable, robotic autostainer (BioGenex i6000 Model, Fremont, CA, USA).

\section{Statistical analyses}

The baseline characteristics between PAD and controls subjects were compared using general linear models for continuous variables and Chi square tests for categorical variables to determine confounders. Confounding variables were covariates in subsequent analyses. For all biological parameters, group differences were determined by analysis of covariance (ANCOVA) and evaluated posthoc by Bonferroni adjusted $\mathrm{t}$ tests. Correlations were assessed by the Pearson test. All statistical analyses were performed with SPSS 20 (IBM, Armonk, NY, USA) using a confidence level of $95 \%$.

\section{Results}

\section{Patient demographics}

Data for CTRL, PAD-II, and PAD-IV patients are presented in Table 1 . On average, PAD-IV patients were 6 years older than PAD-II $(\mathrm{p}=0.047)$ and 8 years older than CTRL ( $\mathrm{p}=0.006)$ patients. More PAD-IV patients had diabetes than PAD-II and CTRL patients $\left(\chi^{2}=5.55\right.$, $\mathrm{p}=0.006$; both $\mathrm{p}<0.05)$. Age and diabetes were treated as covariates in all subsequent analyses.

\section{PAD gastrocnemius specimens exhibited increased collagen deposition with advancing disease stage}

Spectral imaging revealed increased collagen density with higher Fontaine stage, which became diffuse in Stage IV muscle (Fig. 1a). Collagen density and area in PAD muscle was increased between the myofibers and around the lumen of microvessels. The most noticeable pathological change was dense collagenous investment of the microvessels of PAD muscle (arrows). Spectral analysis established increased collagen density $(\mathrm{p}<0.001)$ and area $(\mathrm{p}<0.001)$ at the higher Fontaine stage. Collagen density in the PAD-IV patients $(2708.8 \pm 612.3$ gsu) was 40 and $75 \%$ greater than in PAD-II and CTRL patients, respectively $(1969.9 \pm 277.5$ and $1551.7 \pm 232.8 \mathrm{gsu}$; both $\mathrm{p}<0.001$ ), while collagen density was $25 \%$ greater in PAD-II compared to CTRL ( $\mathrm{p}=0.015$ ) (Fig. 1b). Collagen area was approximately twice as great in PAD-IV $\left(241,179 \pm 133,159 \mathrm{~mm}^{2}\right)$ compared to either PAD-II or CTRL gastrocnemius $\left(109,179 \pm 56,481 \mathrm{~mm}^{2}\right.$ and $118,624 \pm 99,559 \mathrm{~mm}^{2}$; both $\left.\mathrm{p}<0.01\right)$, with no difference between PAD-II and CTRL (Fig. 1c). These data suggest that increased collagen deposition occurs first around 
Table 1 Demographics of study groups

\begin{tabular}{|c|c|c|c|c|}
\hline & CTRL & PAD-II & PAD-IV & $p$ value \\
\hline Number of patients & 20 & 25 & 20 & N/A \\
\hline Mean age (years) & $62.1 \pm 5.40$ & $64.1 \pm 7.80$ & $69.9 \pm 9.40^{*}$ & 0.011 \\
\hline Gender (male/female) & $19 / 1$ & $24 / 1$ & $20 / 0$ & 0.983 \\
\hline Height (m) & $1.75 \pm 0.07$ & $1.76 \pm 0.06$ & $1.77 \pm 0.06$ & 0.576 \\
\hline Weight (kg) & $91.0 \pm 17.4$ & $87.5 \pm 18.8$ & $85.0 \pm 21.1$ & 0.598 \\
\hline Body mass index & $29.9 \pm 6.40$ & $28.2 \pm 5.16$ & $28.4 \pm 6.61$ & 0.593 \\
\hline Obesity $^{\mathrm{a}}(\%)$ & 45 & 36 & 25 & 0.416 \\
\hline Smoking (\%) & 50.0 & 56.0 & 30.0 & 0.202 \\
\hline Diabetes Mellitus (\%) & 25.0 & 20.0 & $65.0^{*}$ & 0.004 \\
\hline Dyslipidemia (\%) & 55.0 & 80.0 & 60.0 & 0.166 \\
\hline Coronary artery disease (\%) & 20.0 & 36.0 & 35.0 & 0.701 \\
\hline Myocardial infraction (\%) & 0.00 & 8.00 & 0.00 & 0.192 \\
\hline Hypertension (\%) & 65.0 & 84.0 & 90.0 & 0.116 \\
\hline Statins Medication (\%) & 70.0 & 84.0 & 65.0 & 0.317 \\
\hline Renal insufficiency ${ }^{\mathrm{b}}(\%)$ & 5.00 & 12.0 & 20.0 & 0.352 \\
\hline Ankle Brachial Index (minimum-maximum) & $1.04 \pm 0.11^{*}(0.79-1.20)$ & $0.55 \pm 0.22^{*}(0.10-0.95)$ & $0.22 \pm 0.13^{*}(0.00-0.44)$ & $<0.001$ \\
\hline
\end{tabular}

CTRL Control subject, PAD-II PAD patient at Fontaine Stage II, PAD-IV PAD patient at Fontaine Stage IV

* $\mathrm{p}<0.05$ compared to each of the other two groups by post-hoc Bonferroni adjusted t tests

a Obesity: Body mass index $>30$

b Renal insufficiency: Creatinine clearance $<60 \mathrm{ml} / \mathrm{min} / 1.73 \mathrm{~m} 2$

c $\mathrm{ABl}$ : data presented as mean \pm standard deviation

microvessels and then expands throughout the extracellular matrix between myofibers and myofascicles as PAD advances.

\section{TGF- $\beta 1$ expression is tightly linked to myofibrosis of PAD gastrocnemius}

QFM imaging localized TGF- $\beta 1$ expression to the vasculature of both CTRL and PAD muscle, with no detectable labeling outside of the vascular walls (Fig. 2a). TGF- $\beta 1$ expression was uniformly low in CTRL muscle and exhibited a progressive increase in PAD-II and PADIV muscle. PAD-IV gastrocnemius had approximately 2.5- and 8-fold greater expression of TGF- $\beta 1$ compared to PAD-II and CTRL patients $(6.56 \pm 3.12$ vs. $2.89 \pm 2.12$ and $0.842 \pm 0.399 \mathrm{gsu}$, respectively; both $\mathrm{p}<0.001$ ), while PAD-II had 3.5-fold more TGF- $\beta 1$ than CTRL $(\mathrm{p}<0.05$; Fig. $2 \mathrm{~b}$ ). Vascular TGF- $\beta 1$ expression positively correlated with collagen density across all subjects $(\mathrm{N}=65)$ in this study ( $r=0.798, \mathrm{p}<0.001$; Fig. 2c). Separate analysis of the PAD patients alone (PAD-II and PAD-IV; $\mathrm{N}=45$ ) and the CTRL subjects alone $(\mathrm{N}=20)$ revealed a significant correlation between TGF- $\beta 1$ and collagen density in the PAD group $(r=0.854, \mathrm{p}<0.001)$, but not in the CTRL group $(r=0.119, \mathrm{p}=0.618)$, indicating that the pathological fibrosis in PAD is driven by TGF- $\beta 1$ expression. Across all subjects $(\mathrm{N}=65)$ in this study, vascular TGF- $\beta 1$ expression increased with decreasing $A B I$ $(\mathrm{r}=-0.694, \mathrm{p}<0.001$; Fig. $2 \mathrm{~d})$, which is an indicator of blood flow (hemodynamic) compromise and increased ischemia of the lower limbs. The relationship between TGF- $\beta 1$ and ABI remained significant when analyzing PAD patients alone $(r=-0.543, \mathrm{p}<0.001)$. These findings identify increased microvascular TGF- $\beta 1$ expression as a characteristic of PAD muscle and establish an association between increased TGF- $\beta 1$ expression and muscle fibrosis and between increased TGF- $\beta 1$ expression and advancing disease stage. Additionally, they show that as the blood flow (and presumably oxygenation) to the leg is decreased by atherosclerotic blockages in the arteries supplying the legs (reflected by decreasing ABI) there is a corresponding increase in the expression of TGF$\beta 1$ expression in the microvessels of the muscles of the affected leg.

\section{Increased expression of TGF- $\beta 1$ is associated with accumulation of fibroblasts and collagen deposition in PAD gastrocnemius}

To further evaluate the concept of a TGF- $\beta 1$ dependent myofibrosis in PAD, we selected three adjacent gastrocnemius sections and stained the first section for collagen with Masson Trichrome, the second for TE-7 positive fibroblasts by immunohistochemistry, and the third for TGF- $\beta 1$ by immunofluorescence. Images from a PADIV patient with significant fibrosis are shown in Fig. 3. 


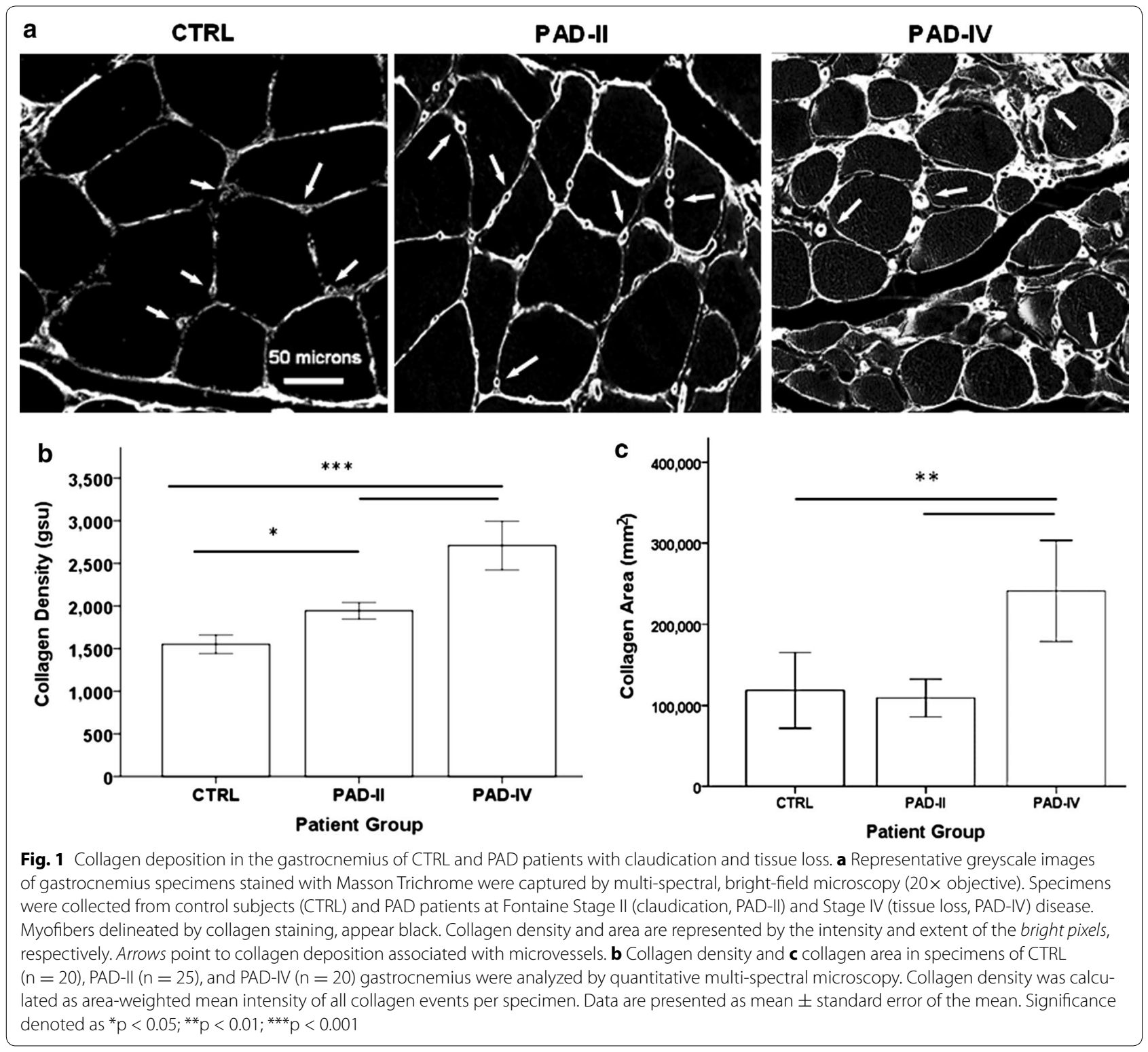

In areas of dense collagen deposition (ellipse, Fig. 3a), we observed a high density of fibroblasts (ellipse, Fig. 3b). This is in contrast to areas containing relatively little collagen (circle, Fig. 3a), which had very few fibroblasts (circle, Fig. 3b). Vessels near the heavily fibrotic areas (high magnification of the square in Fig. 3a) contain a high density of fibroblasts (Fig. 3c) in association with intense TGF- $\beta 1$ labeling (Fig. 3d) and dense adventitial collagen (square, Fig. 3a). These observations are consistent with the pro-fibrotic activity of TGF- $\beta 1$, where TGF- $\beta 1$ activates motile fibroblasts that deposit collagen $(29,30)$, and establish a spatial association of increased TGF$\beta 1$ expression, fibroblast accumulation, and collagen deposition as a characteristic of PAD pathophysiology.
They also further demonstrate that the microvessels of ischemic legs play a key role in the pathophysiology of PAD myofibrosis.

TGF- $\beta 1$ is expressed by vascular smooth muscle cells in the microvessels of PAD gastrocnemius

To determine the specific cellular source of TGF- $\beta 1$ expression in the microvessels of PAD gastrocnemius, we implemented co-localization studies with antibodies for highly specific markers of candidate vascular cells, including endothelial cells, SMC, fibroblasts, macrophages, and T cells (Fig. 4). TGF- $\beta 1$ labeling did not co-localize with CD163 positive macrophages (Fig. 4a), CD3 positive $T$ cells (Fig. 4b), or CD31 positive endothelia (Fig. 4c). TE-7 

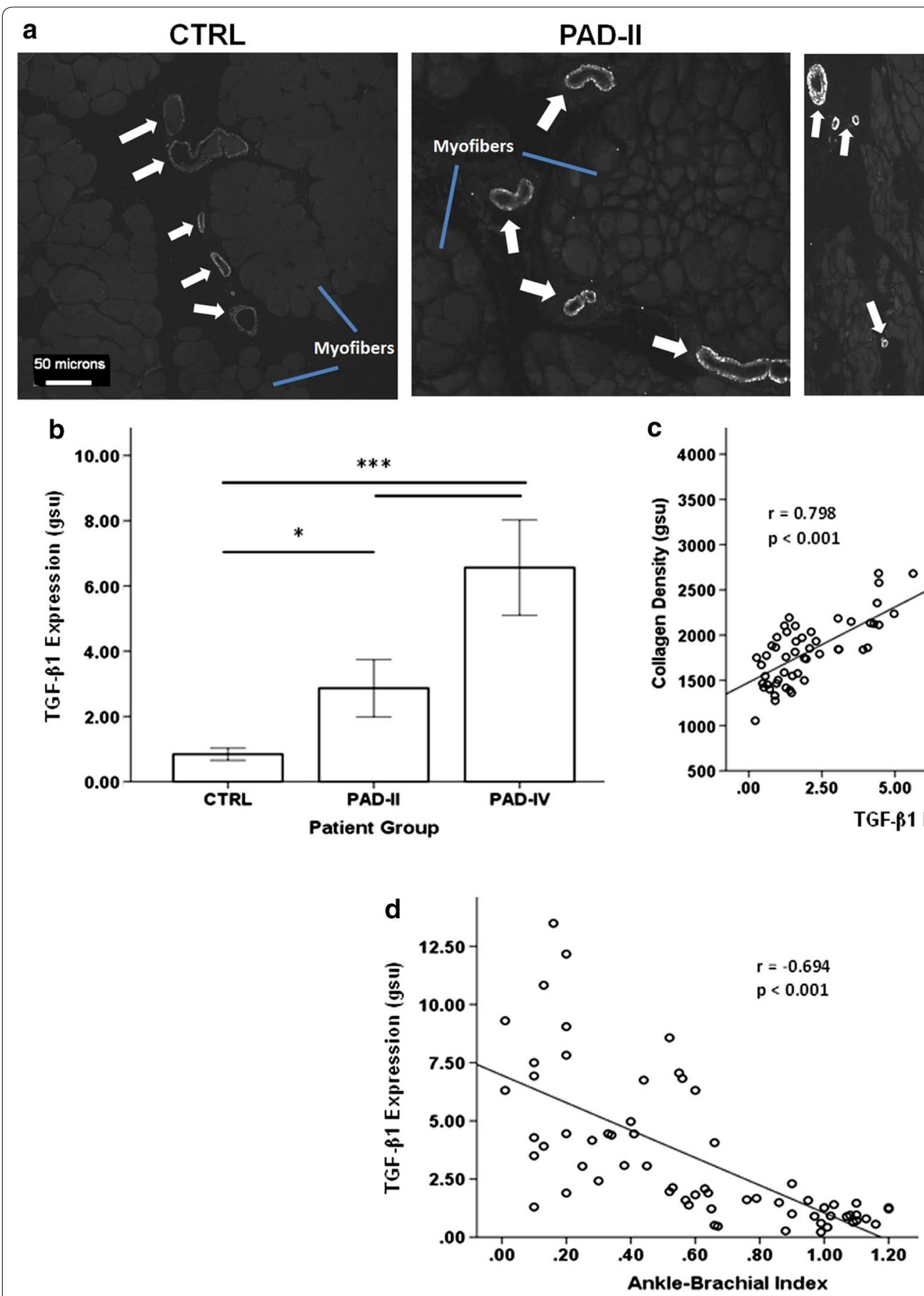

Fig. 2 TGF- $\beta 1$ expression in CTRL and PAD gastrocnemius and its relationship with collagen density and ABI. a Representative greyscale images of gastrocnemius microvessels positive for TGF- $\beta 1$ labeling (arrows) were captured with a wide-field fluorescence microscope (10x objective). Unlabeled myofibers (blue line) appear grey against black background. Specimens collected from control subjects (CTRL) and PAD patients at Fontaine Stage II (claudication, PAD-II) and Stage IV (tissue loss, PAD-IV) disease were labeled with primary antibody specific for TGF- $\beta 1$ and a fluorescent secondary antibody. Both the intensity and extent of TGF- $\beta 1$ labeling were increased in the microvessels of PAD versus CTRL and PAD-IV versus PAD-II specimens. b TGF- $\beta 1$ expression was determined by quantitative fluorescence microscopy and defined as the sum of the products of area and mean pixel intensity of all positive events per microscopic field, normalized to the total area of specimen in the same field. The relationships between normalized TGF- $\beta 1$ expression and collagen density (c), and Ankle-Brachial Index (d) were determined by the Pearson correlation analysis. Data are presented as mean \pm standard error of the mean and significance is denoted as ${ }^{*} p<0.05 ;{ }^{* *} p<0.01 ;{ }^{* * *} p<0.001$ 

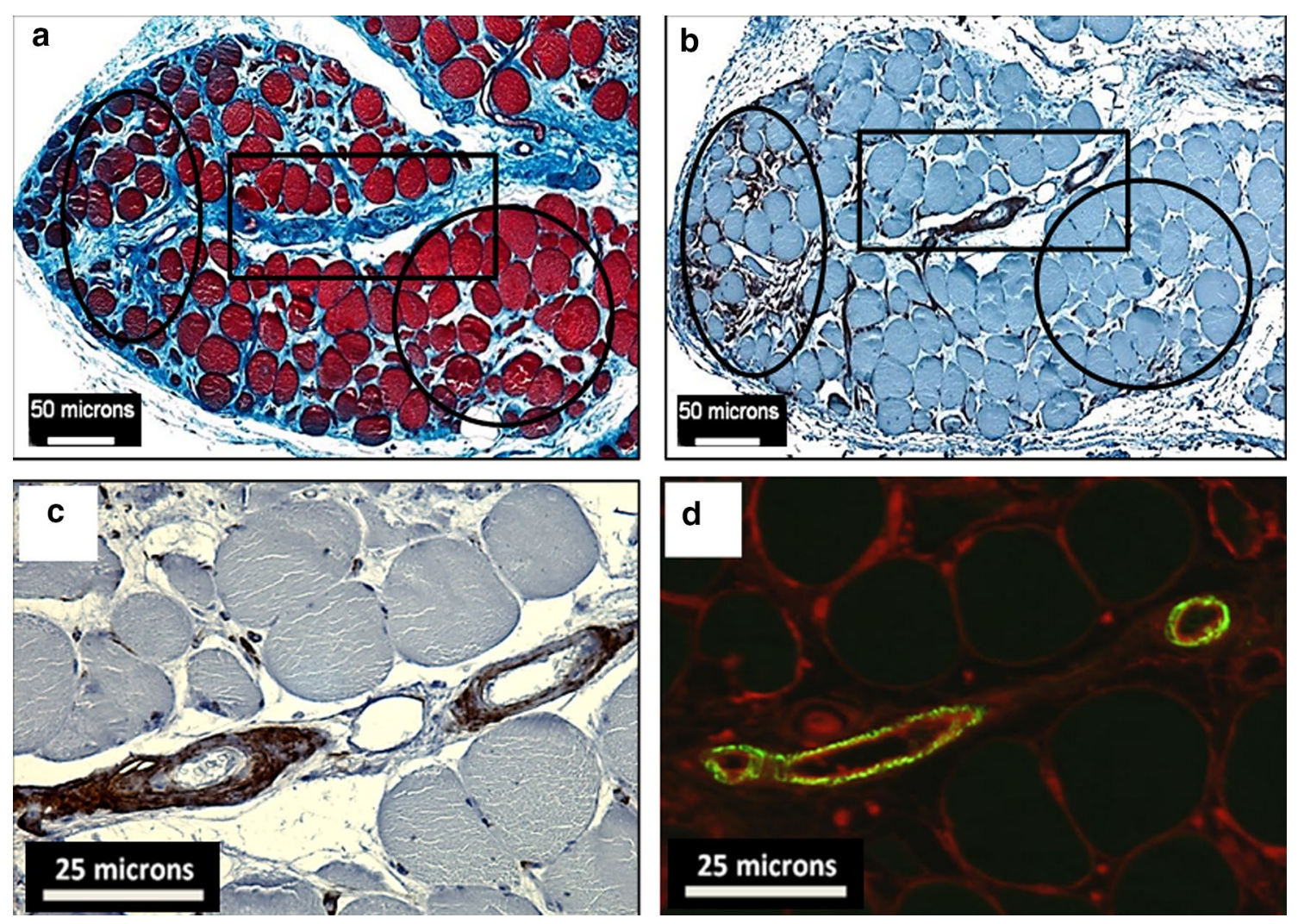

Fig. 3 Association of TGF- $\beta 1$ expression with fibroblast accumulation and collagen deposition in gastrocnemius of PAD patients. All images are of the gastrocnemius of a representative PAD patient who presented with tissue loss. a Masson Trichrome staining reveals highly fibrotic regions (blue labeling) around myofibers (oval) and microvessels (rectangle), and a region with relatively little fibrosis (circle). b A neighboring 4-micron section was labeled by immunohistochemistry with anti-TE-7 antibody, a fibroblast marker which identified fibroblasts in the same three regions of interest. c High magnification of the rectangular region of interest reveals the extent of fibroblast accumulation with microvessels. $\mathbf{d}$ A neighboring section labeled by immunofluorescence for TGF- $\beta 1$ (green fluorescence) shows the intensity and extent of TGF- $\beta 1$ labeling of microvessels within the rectangular region of interest. Wheat Germ Agglutinin (WGA; red fluorescence) labeled membranes and was used to delineate myofibers

positive fibroblasts located in the adventitia were not positive for TGF- $\beta 1$, nor were cells stained with TE-7 in the intima, which may represent endothelial-mesenchymal transition (Fig. 4d). TGF- $\beta 1$ co-localized with cells expressing high molecular weight caldesmon, a marker of SMC that is not found in macrophages or fibroblasts $[46,47]$ (Fig. 4e). Prominent co-localization was observed in rhomboidal-shaped SMC located in the subendothelial region (arrows). This has been reported for other vascular fibrotic pathologies, where SMC are activated toward the pro-fibrotic synthetic phenotype, including in atherosclerotic arterial plaques. Many of the rhomboidal SMC stained positive for Ki-67 in the nuclei (arrowheads; Fig. 4f), indicating they are proliferative and activated. The finding that TGF- $\beta 1$ is derived from locally proliferative SMC rather than immune cells in PAD myofibrosis is novel.

\section{Discussion}

This study established that vascular TGF- $\beta 1$ is strongly associated with myofibrosis during the progression of PAD. Using rigorous quantitative methods, we have shown that vascular expression of TGF- $\beta 1$ and collagen deposition in PAD gastrocnemius increased in parallel with advancing disease severity. Qualitatively, TGF$\beta 1$ production was limited to cells in the walls of the microvessels in affected PAD muscle. In advanced disease, these cells expressing TGF- $\beta 1$ were present at high density and were associated with accumulation of fibroblasts and increased deposition of collagen around the vessels and throughout an expanded interstitium. Our findings suggest that increased vascular TGF- $\beta 1$ induces PAD myofibrosis by activating fibroblasts that proliferate and either stay locally around the microvessels or migrate between myofibers and around myofascicles into 

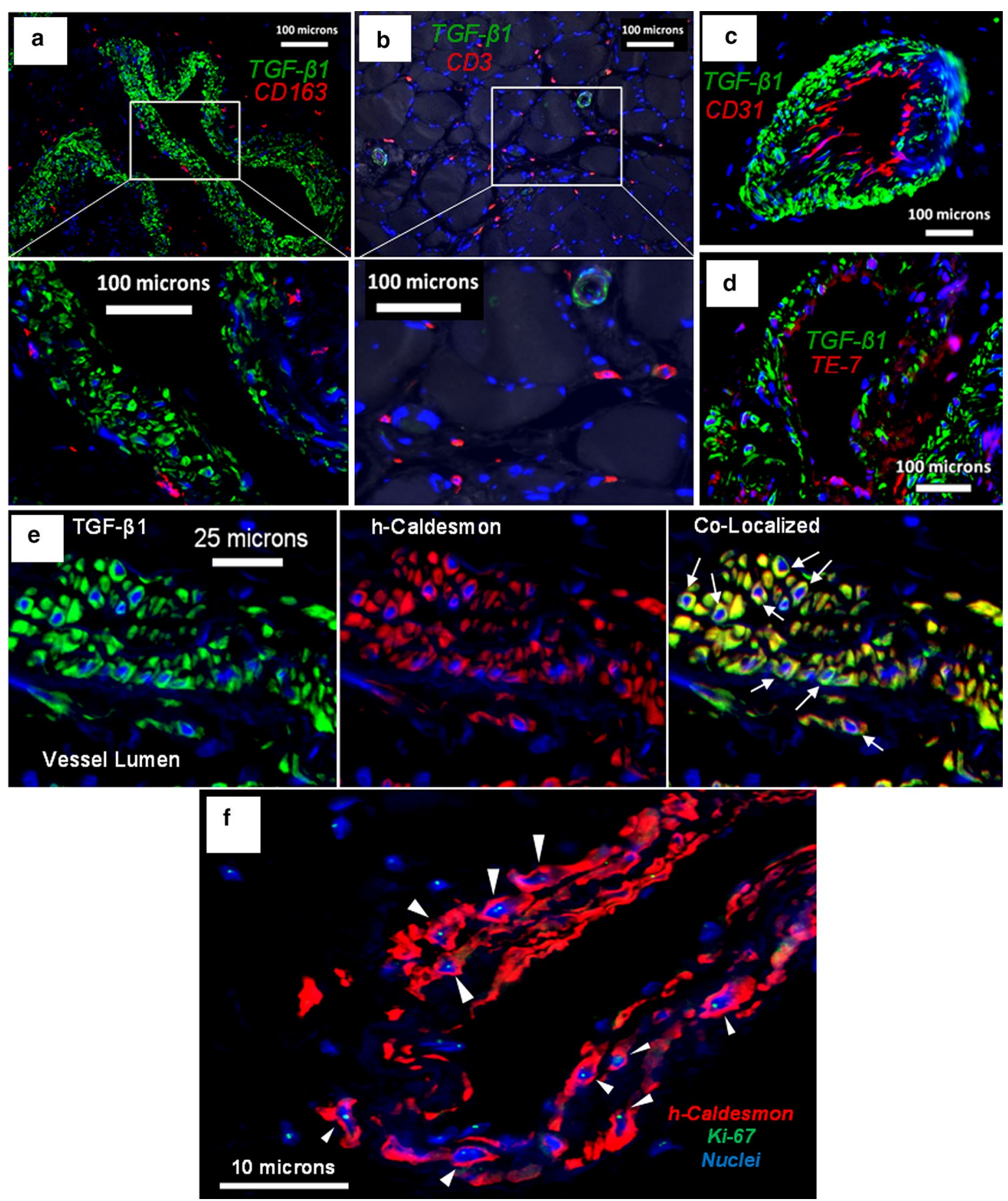

Fig. 4 Evaluation of candidate vascular cells for TGF- $\beta 1$ expression. All immunofluorescence images are from PAD patients who present with tissue loss and are representative of all diseased patients in our study. a TGF- $\beta 1$ labeling (green) does not co-localize with CD163 positive macrophages (red) present at relatively high density in the adventitia of microvessels. High magnification of the boxed region reveals cellular labeling of CD163 around DAPI stained nuclei (b/ue). b TGF- $\beta 1$ labeling (green) does not co-localize with CD3 positive T cells (red) that are located typically around myofibers near microvessels. High magnification of the boxed region reveals cellular labeling of CD3 around DAPI stained nuclei (blue). c TGF- $\beta 1$ labeling (green) does not co-localize with CD31 positive endothelial cells (red) that are characteristically located in the intima of microvessels. $\mathbf{d}$ TGF- $\beta 1$ labeling (green) does not co-localize with TE-7 positive fibroblasts (red). e TGF- $\beta 1$ labeling (green) co-localizes with high molecular weight caldesmon (h-Caldesmon) a specific marker of smooth muscle cells (red). Arrows point to rhomboidal morphology characteristic of secretory SMC. $\mathbf{f}$ The proliferation marker Ki-67 (green) is expressed in nuclei (blue) of h-Caldesmon positive SMC (red) that highly express TGF- $\beta 1$ as determined with a neighboring tissue section 
areas of myofiber degeneration, thus being responsible for the perivascular and endomysial/perimysial fibrosis we and others $[48,49]$ have shown in the affected leg muscles of PAD patients. Accumulation of fibrous tissue in the PAD muscle can affect the muscle both by compromising the function of its myofibers and myofascicles but also by interfering with the function of its microvessels. The fibrosis of microvessels in PAD muscle has been shown by us and others $[48,49]$ to involve both collagenous thickening of the capillaries and muscularization and fibrosis of normally non-muscularized microvessels and is known to impair diffusion of gases and small molecules between the vasculature and end organ parenchyma, probably enhancing the tissue hypoxia and inducing myofiber degeneration in the affected PAD legs. TGF- $\beta 1$ may also be contributing to PAD myopathy by suppressing skeletal muscle regeneration by inducing myoblasts to differentiate into myofibroblasts rather than new myofibers $[21,22]$. This model of PAD myofibrosis is consistent with the contribution of TGF- $\beta 1$ to fibrosis and muscle degeneration seen in other skeletal myopathies [19] and myocardiopathies [50,51].

Another novel finding of this study is that TGF- $\beta 1$ is expressed exclusively by vascular SMC in PAD muscle. Our co-localization studies show conclusively that TGF- $\beta 1$ is produced by vascular SMC and not in the associated fibroblasts, suggesting that these fibroblasts are activated by TGF- $\beta 1$ produced in the SMC. These observations again point to a widely reported mechanism of fibrosis in human fibrotic diseases, in which TGF- $\beta 1$ activates fibroblasts to myofibroblasts. Our co-localization studies also revealed that macrophages and T cells, which are reported to be the source of TGF- $\beta 1$ in other skeletal myofibroses, did not express detectable TGF- $\beta 1$ in PAD muscle $[19,20]$. This is likely due to the different etiology of PAD myofibrosis compared to other skeletal myopathies, of which the muscular dystrophies are the best studied. The primary cause of PAD myopathy is atherosclerotic stenoses and occlusions of large arteries that supply the legs, which produce ischemia and hypoxia in the lower extremities [4]. Over time, chronic hypoxia may damage the muscle myofibers to produce their characteristic degeneration and induce SMC of the microvessels to increase TGF- $\beta 1$ expression and cause myofibrosis. In contrast, muscular dystrophies originate from mutated genes that code for defective skeletal muscle proteins primarily involved in transmitting sarcomeric forces to the extracellular matrix $[19,20]$. In muscular dystrophies, repeated cycles of myofiber degeneration and regeneration induce a chronic inflammatory response that includes TGF- $\beta 1$ production by activated immune cells and produces the myofibrosis. Progressive fibrosis in PAD muscle appears not to be a response to chronic inflammation. We have profiled cytokines in the gastrocnemius of moderately diseased PAD patients and, indeed, did not find a generalized inflammatory signature [18]. The unique cytokine milieu produced by chronic ischemia in PAD gastrocnemius may explain why we did not observe detectable TGF- $\beta 1$ in macrophages and $\mathrm{T}$ cells as is seen, e.g., in muscular dystrophies. Overall, the vascular etiology of PAD myofibrosis points to the possibility that chronic hypoxia of microvessels causes increased expression of TGF- $\beta 1$ by SMC.

In PAD, hypoxic injury may cause transitioning of SMC from a contractile to a synthetic phenotype that is pro-fibrotic. Vascular SMC are normally contractile, with a spindle-shaped morphology and an abundance of contractile proteins that allow them to regulate vessel diameter. Under various pathological conditions marked by hypoxia, SMC can acquire a synthetic phenotype marked by rhomboidal morphology, decreased contractile proteins, and the ability to proliferate, migrate, and deposit collagen [31, 32]. In this study, we found that patients with lower ABI had greater expression of TGF$\beta 1$ across Fontaine Stages of disease, which suggests that hypoxia induces SMC to increase TGF- $\beta 1$ expression. This is supported by our observation that many SMC with increased TGF- $\beta 1$ expression were proliferative and exhibited a rhomboidal morphology that is characteristic of synthetic SMC.

The responses of vascular SMC to hypoxia in the ischemic muscle of PAD patients are likely similar to those in pulmonary arterial hypertension $(\mathrm{PAH})$ and may provide insight into the mechanisms by which TGF- $\beta 1$ expression is increased in PAD muscle. PAH is a condition characterized by substantial thickening of the vascular wall caused by extensive proliferation of SMC that exhibit increased TGF- $\beta 1$ expression and deposition of collagen [34, 35]. Hypoxia alone can stimulate proliferation of human pulmonary SMC in culture [34]. Alternatively, growth factors released by cultured endothelial cells exposed to hypoxia also can stimulate proliferation of SMC [52]. Either or both mechanisms may operate in vivo causing SMC to shift towards a more proliferative, synthetic phenotype in PAH. In PAD gastrocnemius, SMC with intense TGF- $\beta 1$ labeling were observed frequently in the sub-endothelial region, suggesting that hypoxic insult to endothelial cells may stimulate secretion of growth factors that cause SMC to increase TGF$\beta 1$ expression. Given the histopathological similarities between PAH and PAD, future mechanistic studies of PAD myofibrosis should determine whether chronic hypoxia directly induces TGF- $\beta 1$ expression by SMC, and/or activates endothelial cells to increase production of pro-fibrotic factors that cause SMC to increase expression of TGF- $\beta 1$. 
Understanding the mechanism by which chronic hypoxia stimulates SMC production of TGF- $\beta 1$ can lead to development of animal models that recapitulate the myofibrosis observed in patients with PAD. Such models will allow for testing of novel anti-fibrotic therapies that modulate the cellular phenotypes and growth factors released from specific cell types to reduce SMC expression of TGF- $\beta 1$ and myofibrosis. Importantly, we can determine side effects in these animals that may hamper the success of therapeutic strategies. Additionally, limb function can be assessed over time and in relation to changes in myofibrosis.

A limitation of this study is the number of human subjects, however our power calculations indicated enough statistical power to analyze each of the biological parameters. The main barriers against recruitment of additional human subjects are the rigorous inclusion and exclusion criteria used to reduce confounding variables, and the demands of obtaining muscle biopsies. PAD patients included in our study must have had exercise-limiting claudication established by history and direct observation during a screening walk test administered by a vascular surgeon. We enrolled only those patients who did not exhibit concurrent symptoms of heart, lung, musculoskeletal (mainly arthritis), and neurologic (mainly back pain and sciatica) ailments that would affect their walking ability and their performance in the screening walk test. Furthermore, we excluded individuals with (1) asymptomatic PAD, i.e., patients with occlusive arterial disease who do not have claudication symptoms or tissue loss/gangrene, (2) acute lower extremity ischemic events secondary to thromboembolic disease or acute trauma, and (3) exercise capacity limited by conditions other than claudication including leg (joint or musculoskeletal and neurologic) and systemic (heart and lung disease) pathology. The invasiveness of a muscle biopsy and the time required often discourages participation of PAD patients and control subjects especially when the muscle biopsy cannot be performed during their routine care (at the time of a leg operation) which was frequently the case for most PAD-II and control patients. With the 65 patients recruited, post-hoc power sample analysis using the data (mean and SD) for collagen density and area, and TGF$\beta 1$ expression, revealed adequate statistical power. Power sample analysis for an ANCOVA of TGF- $\beta 1$ expression demonstrated that a total sample size of $\mathrm{N}=65(20,25$ and 20 subjects per group) assured at least $99 \%$ power to detect differences between groups adjusting for two covariates with a conservative $R^{2}$ value of 0.20 . Similarly, for an ANCOVA of collagen density and area, our sample size assured at least 99 and $93 \%$ power, respectively, to detect differences between the groups adjusting for two covariates with a conservative $R^{2}$ value of 0.20 . The power and sample size determination package PASS (PASS, Number Cruncher Statistical Systems, Kaysville, UT) was used for the analysis.

An additional limitation is the correlational nature of the study, but the high quality human data that we have presented for PAD myofibrosis provide a basis for future mechanistic studies, development of disease models, and improved therapies and prognosis. In this study, we established an association between ischemia and increased TGF- $\beta 1$ production by microvascular SMC. We have identified microvascular SMC in PAD muscle as the exclusive producer of TGF- $\beta 1$, making it a specific target for anti-fibrotic therapies for PAD. Candidate therapeutic drugs will be those capable of shifting the pro-fibrotic synthetic phenotype of SMC back to the contractile form to decrease TGF- $\beta 1$ expression and prevent or slow down the progression of PAD myofibrosis. Moreover, TGF- $\beta 1$ production by SMC may be a potential biomarker for determining the efficacy of therapeutics, including anti-fibrotic interventions. Finally, our finding of the progressive worsening of myofibrosis with advancing Fontaine Stage in PAD suggests that the patients who are optimal for anti-fibrotic intervention are those with moderate disease, since (1) a relatively small proportion of vascular SMC express TGF- $\beta 1$, (2) fibroblasts are present at a relatively low density, and (3) collagen deposition is largely limited to microvessels with little expansion into the interstitium.

\section{Conclusions}

Fibrosis is often viewed as an adaptive response to injury and tissue degeneration, but as we have shown in this study, can be part of the pathophysiology of chronic disease. We have established that increased expression of TGF- $\beta 1$ by microvascular SMC in the gastrocnemius of PAD patients correlates with Fontaine Stage and increasing collagen deposition. The pattern of vascular TGF- $\beta 1$ expression, fibroblast accumulation, and collagen deposition points to pathological changes in microvessels as the immediate cause of PAD myofibrosis. The contribution of hypoxia was suggested by a strong negative correlation between ABI and vascular TGF- $\beta 1$ expression and presence of locally proliferative rhomboidal SMC in microvessels of PAD gastrocnemius that are indicative of the pro-fibrotic synthetic phenotype of SMC known to be induced by hypoxia. Collectively, these findings provide insight into the development of PAD myofibrosis and direction for future mechanistic studies, and consequently, a basis for improved diagnosis and treatment for patients with PAD. 


\section{Additional file}

Additional file 1. Validation of Measurements of Collagen Deposition and TGF- $\beta 1$ Expression. This supplement provides detailed methodologies and the results for validation studies of multi-spectral analysis and quantitative fluorescence microscopy and for intrasession and intersession reliability studies of collagen deposition and TGF- $\beta 1$ expression measurements.

\section{Abbreviations}

PAD: peripheral artery disease; TGF- $\beta 1$ : transforming growth factor beta-1; SMC: smooth muscle cells; $\mathrm{ABI}$ : ankle brachial index.

\section{Authors' contributions}

DMH contributed to the conception and design of all experiments, the acquisition, analysis, and interpretation of all data, and drafted the manuscript. LCC contributed to the acquisition of multi-spectral and quantitative fluorescence microscopy data, the design of the analytical methods in Image Pro Plus software, and all validation studies. PK contributed to the conception and design of quantitative fluorescence microscopy and co-localization experiments, the Masson Trichrome staining, and statistical analyses. SAS and ZZ contributed to the processing of biopsy samples and to the execution and interpretation of the validation studies. $\mathrm{MH}$ contributed to the acquisition of multispectral and quantitative fluorescence microscopy data, statistical analysis, and interpretation of data. HKD contributed to the recruitment and evaluation of patients, performance of the biopsy procedures, processing of muscle samples and analysis of the clinical data. IIP contributed to the recruitment and evaluation of patients, performance of the biopsy procedures, processing of muscle samples, analysis and interpretation of all data, and critical revision of the manuscript; GPC contributed to the conception and design of all experiments, analysis and interpretation of all data, and drafting and critical revision of the manuscript. All authors read and approved the final manuscript.

\section{Author details}

${ }^{1}$ Department of Surgery, University of Nebraska Medical Center, Omaha, NE, USA. ${ }^{2}$ Department of Cellular and Integrative Physiology, University of Nebraska Medical Center, Omaha, NE, USA. ${ }^{3}$ Department of Surgery and VA Research Service, VA Nebraska-Western lowa Health Care System, Omaha, NE, USA. ${ }^{4} 987690$ Nebraska Medical Center, Omaha, NE 68198-7690, USA. ${ }^{5} 983280$ Nebraska Medical Center, Omaha, NE 68198-7690, USA.

\section{Acknowledgements}

The authors gratefully acknowledge Karen Dulany from the University of Nebraska Medical Center for preparing microscopic slide sections of tissue biopsy samples. This work was supported by NIH Grant R01AG034995 and by the Charles and Mary Heider Fund for Excellence in Vascular Surgery. Furthermore, this material is the result of work supported with resources and the use of facilities at the Veterans Affair Nebraska-Western lowa Health Care System.

\section{Competing interests}

There are no financial or non-financial competing interests to disclose for any of the authors.

Received: 28 July 2015 Accepted: 6 January 2016

Published online: 04 February 2016

\section{References}

1. Norgren L, Hiatt WR, Dormandy JA, Nehler MR, Harris KA, Fowkes FG, et al. Inter-society consensus for the management of peripheral arterial disease (TASC II). Eur J Vasc Endovasc Surg. 2007;33(Suppl 1):S1-75.

2. Selvin E, Erlinger TP. Prevalence of and risk factors for peripheral arterial disease in the United States: results from the National Health and Nutrition Examination Survey, 1999-2000. Circulation. 2004;110(6):738-43.

3. Shammas NW. Epidemiology, classification, and modifiable risk factors of peripheral arterial disease. Vasc Health Risk Manag. 2007;3(2):229-34.
4. Olin JW, Sealove BA. Peripheral artery disease: current insight into the disease and its diagnosis and management. Mayo Clin Proc. 2010;85(7):678-92.

5. Pipinos II, Judge AR, Selsby JT, Zhu Z, Swanson SA, Nella AA, et al. The myopathy of peripheral arterial occlusive disease: part 1. Functional and histomorphological changes and evidence for mitochondrial dysfunction. Vasc Endovascular Surg. 2008;41(6):481-9.

6. Pipinos II, Judge AR, Selsby JT, Zhu Z, Swanson SA, Nella AA, et al. The myopathy of peripheral arterial occlusive disease: Part 2. Oxidative stress, neuropathy, and shift in muscle fiber type. Vasc Endovascular Surg. 2008:42(2):101-12.

7. Brass EP, Hiatt WR. Acquired skeletal muscle metabolic myopathy in atherosclerotic peripheral arterial disease. Vasc Med. 2000;5(1):55-9.

8. Pipinos II, Judge AR, Zhu Z, Selsby JT, Swanson SA, Johanning JM, et al. Mitochondrial defects and oxidative damage in patients with peripheral arterial disease. Free Radic Biol Med. 2006;41(2):262-9.

9. Koutakis P, Johanning JM, Haynatzki GR, Myers SA, Stergiou N, Longo GM, et al. Abnormal joint powers before and after the onset of claudication symptoms. J Vasc Surg. 2010;52(2):340-7.

10. Gardner AW, Killewich LA. Lack of functional benefits following infrainguinal bypass in peripheral arterial occlusive disease patients. Vasc Med. 2001;6(1):9-14

11. Regensteiner JG, Wolfel EE, Brass EP, Carry MR, Ringel SP, Hargarten ME, et al. Chronic changes in skeletal muscle histology and function in peripheral arterial disease. Circulation. 1993;87(2):413-21.

12. McDermott MM, Liu K, Tian L, Guralnik JM, Criqui MH, Liao Y, et al. Calf muscle characteristics, strength measures, and mortality in peripheral arterial disease: a longitudinal study. J Am Coll Cardiol. 2012;59(13):1159-67.

13. McDermott MM, Ferrucci L, Guralnik J, Tian L, Liu K, Hoff F, et al. Pathophysiological changes in calf muscle predict mobility loss at 2-year follow-up in men and women with peripheral arterial disease. Circulation. 2009;120(12):1048-55.

14. Farinon AM, Marbini A, Gemignani F, Govoni E, Bragaglia MM, Sianesi $M$, et al. Skeletal muscle and peripheral nerve changes caused by chronic arterial insufficiency-significance and clinical correlationshistological, histochemical and ultrastructural study. Clin Neuropathol. 1984;3(6):240-52

15. Hedberg B, Angquist KA, Henriksson-Larsen K, Sjostrom M. Fibre loss and distribution in skeletal muscle from patients with severe peripheral arterial insufficiency. Eur J Vasc Surg. 1989;3(4):315-22.

16. Cluff K, Miserlis D, Naganathan GK, Pipinos II, Koutakis P, Samal A, et al. Morphometric analysis of gastrocnemius muscle biopsies from patients with peripheral arterial disease: objective grading of muscle degeneration. Am J Physiol Regul Integr Comp Physiol. 2013;305(3):R291-9.

17. Weiss DJ, Casale GP, Koutakis P, Nella AA, Swanson SA, Zhu Z, et al. Oxidative damage and myofiber degeneration in the gastrocnemius of patients with peripheral arterial disease. J Transl Med. 2013;11(1):230. doi:10.1186/1479-5876-11-230.

18. Thompson JR, Casale GP, Swanson SA, Koutakis P, Kim K, Papoutsi E, et al. Quantification of cytokines in the gastrocnemius and serum of claudicating patients with peripheral arterial disease. J Surg Res. 2013;179(2):281.

19. Serrano AL, Mann CJ, Vidal B, Ardite E, Perdiguero E, Munoz-Canoves P. Cellular and molecular mechanisms regulating fibrosis in skeletal muscle repair and disease. Curr Top Dev Biol. 2011;96:167-201.

20. Mann CJ, Perdiguero E, Kharraz Y, Aguilar S, Pessina P, Serrano AL, et al. Aberrant repair and fibrosis development in skeletal muscle. Skelet Muscle. 2011;1(1):21. doi:10.1186/2044-5040-1-21.

21. Levy BI, Schiffrin EL, Mourad JJ, Agostini D, Vicaut E, Safar ME, et al. Impaired tissue perfusion: a pathology common to hypertension, obesity, and diabetes mellitus. Circulation. 2008;118(9):968-76.

22. Dai Z, Aoki T, Fukumoto Y, Shimokawa H. Coronary perivascular fibrosis is associated with impairment of coronary blood flow in patients with nonischemic heart failure. J Cardiol. 2012;60(5):416-21.

23. Wynn TA, Ramalingam TR. Mechanisms of fibrosis: therapeutic translation for fibrotic disease. Nat Med. 2012;18(7):1028-40.

24. Ostriker A, Horita HN, Poczobutt J, Weiser-Evans MC, Nemenoff RA Vascular smooth muscle cell-derived transforming growth factor-beta promotes maturation of activated, neointima lesion-like macrophages. Arterioscler Thromb Vasc Biol. 2014;34(4):877-86. 
25. Gibbons GH, Pratt RE, Dzau VJ. Vascular smooth muscle cell hypertrophy vs. hyperplasia. Autocrine transforming growth factor-beta 1 expression determines growth response to angiotensin II. J Clin Invest. 1992;90(2):456-61.

26. Douillet CD, Velarde V, Christopher JT, Mayfield RK, Trojanowska ME, Jaffa AA. Mechanisms by which bradykinin promotes fibrosis in vascular smooth muscle cells: role of TGF-beta and MAPK. Am J Physiol Heart Circ Physiol. 2000;279(6):H2829-37

27. Lan $T H$, Huang $X Q$, Tan HM. Vascular fibrosis in atherosclerosis. Cardiovasc Pathol. 2013;22(5):401-7.

28. Ruiz-Ortega M, Rodriguez-Vita J, Sanchez-Lopez E, Carvajal G, Egido J. TGF-beta signaling in vascular fibrosis. Cardiovasc Res. 2007;74(2):196-206.

29. Verrecchia F, Mauviel A. Transforming growth factor-beta and fibrosis. World J Gastroenterol. 2007;13(22):3056-62.

30. Biernacka A, Dobaczewski M, Frangogiannis NG. TGF-beta signaling in fibrosis. Growth Factors. 2011;29(5):196-202.

31. Rensen SS, Doevendans PA, van Eys GJ. Regulation and characteristics of vascular smooth muscle cell phenotypic diversity. Neth Heart J. 2007;15(3):100-8

32. Fisher SA. Vascular smooth muscle phenotypic diversity and function. Physiol Genomics. 2010;42A(3):169-87.

33. Gomez D, Owens GK. Smooth muscle cell phenotypic switching in atherosclerosis. Cardiovasc Res. 2012;95(2):156-64.

34. Ismail S, Sturrock A, Wu P, Cahill B, Norman K, Huecksteadt T, et al. NOX4 mediates hypoxia-induced proliferation of human pulmonary artery smooth muscle cells: the role of autocrine production of transforming growth factor- $\beta 1$ and insulin-like growth factor binding protein-3. Am J Physiol Lung Cell Mol Physiol. 2009;296(3):L489-99.

35. Sturrock A, Cahill B, Norman K, Huecksteadt TP, Hill K, Sanders K, et al. Transforming growth factor-beta1 induces Nox4 NAD(P)H oxidase and reactive oxygen species-dependent proliferation in human pulmonary artery smooth muscle cells. Am J Physiol Lung Cell Mol Physiol. 2006;290(4):L661-73.

36. Graham KM, Singh R, Millman G, Malnassy G, Gatti F, Bruemmer K, et al. Excessive collagen accumulation in dystrophic ( $\mathrm{mdx}$ ) respiratory musculature is independent of enhanced activation of the NF-kappaB pathway. J Neurol Sci. 2010;294(1-2):43-50.

37. Huang D, Casale GP, Tian J, Wehbi NK, Abrahams NA, Kaleem Z, et al. Quantitative fluorescence imaging analysis for cancer biomarker discovery: application to beta-catenin in archived prostate specimens. Cancer Epidemiol Biomark Prev. 2007;16(7):1371-81.

38. Richardson T, McCanse W, Casale GP, Huang D, Tian J, Elkahwaji JE, et al. Tissue-based quantification of 8-hydroxy-2'-deoxyguanosine in human prostate biopsies using quantitative fluorescence imaging analysis. Urology. 2009;74(5):1174-9.

39. Huang D, Casale GP, Tian J, Lele SM, Pisarev VM, Simpson MA, et al. Udpglucose dehydrogenase as a novel field-specific candidate biomarker of prostate cancer. Int J Cancer. 2010;126(2):315-27.
40. Koutakis P, Miserlis D, Myers SA, Kim JK, Zhu Z, Papoutsi E, et al. Abnormal accumulation of desmin in gastrocnemius myofibers of patients with peripheral artery disease: associations with altered myofiber morphology and density, mitochondrial dysfunction and impaired limb function. J Histochem Cytochem. 2015;63(4):256-69.

41. Koutakis P, Weiss DJ, Miserlis D, Shostrom VK, Papoutsi E, Ha DM, et al. Oxidative damage in the gastrocnemius of patients with peripheral artery disease is myofiber type selective. Redox Biol. 2014;19(2):921-8.

42. Kim JK, Zhu Z, Casale G, Koutakis P, McComb RD, Swanson S, et al. Human enterovirus in the gastrocnemius of patients with peripheral arterial disease. J Am Heart Assoc. 2013;2(4):e000082.

43. Pilling D, Fan T, Huang D, Kaul B, Gomer RH. Identification of markers that distinguish monocyte-derived fibrocytes from monocytes, macrophages, and fibroblasts. PLoS One. 2009;4(10):e7475.

44. Goodpaster T, Legesse-Miller A, Hameed MR, Aisner SC, RandolphHabecker J, Coller HA. An immunohistochemical method for identifying fibroblasts in formalin-fixed, paraffin-embedded tissue. J Histochem Cytochem. 2008;56(4):347-58.

45. Agley CC, Rowlerson AM, Velloso CP, Lazarus NL, Harridge SD. Isolation and quantitative immunocytochemical characterization of primary myogenic cells and fibroblasts from human skeletal muscle. J Vis Exp. 2015;. doi:10.3791/52049.

46. Lazard D, Sastre X, Frid MG, Glukhova MA, Thiery JP, Koteliansky VE. Expression of smooth muscle-specific proteins in myoepithelium and stromal myofibroblasts of normal and malignant human breast tissue. Proc Natl Acad Sci USA. 1993;90(3):999-1003.

47. Watanabe K, Tajino T, Sekiguchi M, Suzuki T. h-Caldesmon as a specific marker for smooth muscle tumors. Comparison with other smooth muscle markers in bone tumors. Am J Clin Pathol. 2000;113(5):663-8.

48. Ho TK, Rajkumar V, Black CM, Abraham DJ, Baker DM. Increased angiogenic response but deficient arteriolization and abnormal microvessel ultrastructure in critical leg ischaemia. Br J Surg. 2006;93(11):1368-76.

49. Baum O, Djonov V, Ganster M, Widmer M, Baumgartner I. Arteriolization of capillaries and FGF-2 upregulation in skeletal muscles of patients with chronic peripheral arterial disease. Microcirculation. 2005;12(6):527-37.

50. Dobaczewski M, Chen W, Frangogiannis NG. Transforming growth factor (TGF)-beta signaling in cardiac remodeling. J Mol Cell Cardiol. 2011;51(4):600-6.

51. Kong P, Christia P, Frangogiannis NG. The pathogenesis of cardiac fibrosis. Cell Mol Life Sci. 2014;71(4):549-74.

52. Chanakira A, Dutta R, Charboneau R, Barke R, Santilli SM, Roy S. Hypoxia differentially regulates arterial and venous smooth muscle cell proliferation via PDGFR-beta and VEGFR-2 expression. Am J Physiol Heart Circ Physiol. 2012;302(5):H1173-84

\section{Submit your next manuscript to BioMed Central and we will help you at every step:}

- We accept pre-submission inquiries

- Our selector tool helps you to find the most relevant journal

- We provide round the clock customer support

- Convenient online submission

- Thorough peer review

- Inclusion in PubMed and all major indexing services

- Maximum visibility for your research

Submit your manuscript at www.biomedcentral.com/submit
Ciomed Central 